\title{
Body mass index at the crossroads of osteoporosis and type 2 diabetes
}

\author{
Jeonghoon $\mathrm{Ha}^{1}$ and Ki-Hyun Baek ${ }^{2}$
}

\author{
${ }^{1}$ Division of Endocrinology and \\ Metabolism, Department of \\ Internal Medicine, Seoul St. \\ Mary's Hospital, College of \\ Medicine, The Catholic University \\ of Korea, Seoul; ${ }^{2}$ Division of \\ Endocrinology and Metabolism, \\ Department of Internal Medicine, \\ Yeouido St. Mary's Hospital, \\ College of Medicine, The Catholic \\ University of Korea, Seoul, Korea
}

Received: October 20, 2019 Accepted: October 26, 2019

\section{Correspondence to}

Ki-Hyun Baek, M.D.

Division of Endocrinology and Metabolism, Department of Internal Medicine, Yeouido St. Mary's Hospital, College of Medicine, The Catholic University of Korea, 10 63-ro, Yeongdeungpo-gu, Seoul o7345, Korea

Tel: $+82-2-3779-1400$

Fax: +82-2-780-3132

E-mail: drbkh@catholic.ac.kr https://orcid.org/0000-00024097-1077

\section{See Article on Page 1432-1442}

Body mass index (BMI) is used to identify obesity, and higher BMI is associated with various comorbidities including type 2 diabetes, osteoarthritis, cardiovascular disease (CVD), and sleep apnea [1]. The risk of unhealthy conditions increases as BMI exceeds cutoff points, whereas intentional weight loss reverses the risk. As such, BMI, an indicator of obesity, has important clinical utility in predicting and monitoring type 2 diabetes and CVD risk factors. With regard to bone mass, BMI shows a completely different effect; high BMI improves bone mineral density (BMD) and low BMI is a well-documented risk factor for osteoporotic fracture [2]. Further, several studies have demonstrated that high BMI appears to have a protective effect on bone mass, whereas low BMI and sudden loss of body mass lead to a reduction in bone mass $[3,4]$. Therefore, BMI is always at the crossroads of osteoporosis and type 2 diabetes.

Lee et al. [5] determined the optimal BMI for reducing the risk of both osteoporosis and type 2 diabetes based on Korea's nationwide population data. In this study, the authors found that a BMI of 23.0 to $24.9 \mathrm{~kg} / \mathrm{m}^{2}$ is the optimal range for minimizing the risk of both type 2 diabetes and osteoporo- sis by analyzing 8,756 Korean subjects with a mean age of $63.5 \pm 9.4$ years. If the $\mathrm{BMI}$ is higher than this range, the risk of osteoporosis is reduced, but the risk of type 2 diabetes increases. If the BMI is lower than this range, the opposite situation occurs. Bombelli et al. [6] reported that an increase in BMI of $1 \mathrm{~kg} / \mathrm{m}^{2}$ increases the risk of type 2 diabetes by $8.4 \%$. In contrast, a $1 \mathrm{~kg} / \mathrm{m}^{2}$ reduction in BMI is associated with a 1.37-fold reduction (95\% confidence interval, 1.12 to 1.68 ) in the risk of type 2 diabetes among individuals with a BMI $<25 \mathrm{~kg} / \mathrm{m}^{2}$ [7]. A low BMI is an important and modifiable risk factor for the development of osteoporosis and osteoporotic fracture $[3,8]$. As BMI increases by $1 \mathrm{~kg} / \mathrm{m}^{2}$, the risk of osteoporosis in men and women appears to be reduced by $28 \%$ and $13 \%$, respectively [5].

Clinically, osteoporosis should take into account the following situations: the risk of fracture, in addition to osteoporosis diagnosed as BMD, has a significant clinical value; BMD and fracture risk do not always coincide; and in obese or diabetic patients, the BMD may not sufficiently reflect the patient's overall bone condition. The World Health Organization defines osteoporosis as a "progressive systemic skeletal disease characterized by low bone mass and microarchitectural deterioration of bone tissue, with a consequent in- 
crease in bone fragility and susceptibility to fracture" [9]. BMD measured by dual-energy $\mathrm{X}$-ray absorptiometry is a definitive tool for the diagnosis of osteoporosis, and low BMD is strongly associated with an increased risk of fracture. However, independent contributors to the risk of fracture such as age, BMI, or history of smoking or steroid use can also be used to identify subjects at high risk of fracture. Although BMD is relatively high in obese persons, it does not always favorably reduce the risk of fracture. It is generally believed that there is a positive correlation between BMD and BMI, and mechanical loading is a possible explanation for the higher $\mathrm{BMD}$ in obese persons.

Areal BMD measurement may be inaccurate in subjects with central obesity. Kim et al. [10] reported that lumbar spine BMD showed a negative correlation with waist-hip ratio, a representative marker for visceral fat. Moreover, the data suggested that obesity is not entirely protective against fracture. A study from a Fracture Liaison Service by Premaor et al. [11] found an unexpectedly high prevalence of obesity in postmenopausal women presenting with low-trauma fractures. Available evidence supports the lower risk of vertebral fracture; however, the risk of non-vertebral fractures is somewhat increased in obese persons. When diabetes and obesity are linked, the situation becomes more complicated. While most patients with type 2 diabetes are obese and their BMD may be increased, large-scale epidemiological data show that the risk of fracture at all sites is higher in those with type 2 diabetes than in the healthy population [12].

Osteoporosis and type 2 diabetes, representative chronic diseases, are increasing in prevalence worldwide; thus, the proper management of both diseases is becoming increasingly important. BMD is a useful method for diagnosing osteoporosis and reflects the risk of fracture; however, it is somewhat unreasonable to define the clinical importance of osteoporosis only according to BMD, without considering the risk of fracture. In obese patients with type 2 diabetes, it is questionable whether higher BMD is definitively associated with a lower risk of fracture. In this sense, the results would have been more convincing if the risk of fracture was considered in addition to the existing analysis.

Despite certain limitations, the current study is the first to demonstrate an appropriate BMI range for min- imizing the risk of osteoporosis and type 2 diabetes in the Korean population. This study has a strong enlightening purpose to maintain an appropriate weight to keep bones healthy and prevent type 2 diabetes. Clinicians should consider the proper BMI range that can lower the risk of both osteoporosis and type 2 diabetes. The authors of the present study seem to suggest that an ideal BMI should remain at a crossroads.

\section{Conflict of interest}

No potential conflict of interest relevant to this article was reported.

\section{REFERENCES}

1. Williams EP, Mesidor M, Winters K, Dubbert PM, Wyatt SB. Overweight and obesity: prevalence, consequences, and causes of a growing public health problem. Curr Obes Rep 2015;4:363-370.

2. Cummings SR, Nevitt MC, Browner WS, et al. Risk factors for hip fracture in white women. Study of Osteoporotic Fractures Research Group. N Engl J Med 1995;332:767-773.

3. Ravn P, Cizza G, Bjarnason NH, et al. Low body mass index is an important risk factor for low bone mass and increased bone loss in early postmenopausal women. Early Postmenopausal Intervention Cohort (EPIC) study group. J Bone Miner Res 1999;14:1622-1627.

4. Joakimsen RM, Fonnebo V, Magnus JH, Tollan A, Sogaard AJ. The Tromso Study: body height, body mass index and fractures. Osteoporos Int 1998;8:436-442.

5. Lee JH, Kim JH, Hong AR, Kim SW, Shin CS. Optimal body mass index for minimizing the risk for osteoporosis and type 2 diabetes. Korean J Intern Med 2020;35:14321442.

6. Bombelli M, Facchetti R, Sega R, et al. Impact of body mass index and waist circumference on the long-term risk of diabetes mellitus, hypertension, and cardiac organ damage. Hypertension 2011;58:1029-1035.

7. Wainberg M, Mahajan A, Kundaje A, et al. Homogeneity in the association of body mass index with type 2 diabetes across the UK Biobank: a Mendelian randomization study. PLoS Med 2019;16:e1002982.

8. Espallargues M, Sampietro-Colom L, Estrada MD, et al. Identifying bone-mass-related risk factors for fracture to guide bone densitometry measurements: a systematic 
review of the literature. Osteoporos Int 2001;12:811-822.

9. Kanis JA, Melton LJ 3rd, Christiansen C, Johnston CC, Khaltaev N. The diagnosis of osteoporosis. J Bone Miner Res 1994;9:1137-1141.

10. Kim CJ, Oh KW, Rhee EJ, et al. Relationship between body composition and bone mineral density (BMD) in perimenopausal Korean women. Clin Endocrinol (Oxf)
2009;71:18-26.

11. Premaor MO, Pilbrow L, Tonkin C, Parker RA, Compston J. Obesity and fractures in postmenopausal women. J Bone Miner Res 2010;25:292-297.

12. Vestergaard P. Discrepancies in bone mineral density and fracture risk in patients with type 1 and type 2 diabetes: a meta-analysis. Osteoporos Int 2007;18:427-444. 